\title{
Updated Search for Electron Antineutrino Appearance at MiniBooNE
}

\author{
E. D. Zimmerman \\ University of Colorado, Boulder, Colorado 80309 \\ For the MiniBooNE Collaboration
}

\begin{abstract}
The MiniBooNE experiment at Fermilab has updated its search for $\bar{v}_{\mu} \rightarrow \bar{v}_{e}$ oscillations with data collected through May 2011. This represents a statistics increase of 52\% over the result published in 2010. The data favor LSND-like oscillations over a background-only hypothesis at the $91.1 \%$ confidence level. While the new result remains equally consistent with LSND, the compatibility with the background-only hypothesis is improved. An excess of $38.6 \pm 18.5 v_{e}$-like events below $475 \mathrm{MeV}$ is observed, consistent with the observation of such an excess in neutrino mode.
\end{abstract}

Keywords: Antineutrino oscillations, LSND, MiniBooNE

PACS: $14.60 . \mathrm{Pq}, 14.60 . \mathrm{St}$

\section{MINIBOONE}

The MiniBooNE experiment (E898/944) at Fermi National Accelerator Laboratory is a short-baseline neutrino oscillation experiment whose main purpose is to test the LSND oscillation results [1]. MiniBooNE uses an $8 \mathrm{GeV}$ proton beam from the Fermilab Booster to produce pions, which then decay in flight to produce a nearly pure $v_{\mu}$ flux at a mineral oil Cherenkov detector $500 \mathrm{~m}$ away. The detector uses Cherenkov ring shape information to distinguish charged-current $v_{\mu}\left(\bar{v}_{\mu}\right)$ from $v_{e}\left(\bar{v}_{e}\right)$ interactions, searching for an excess of $v_{e}$ which would indicate oscillations. Data collection began in late 2002 with the beam configured to produce neutrinos; since 2007 most operations have been with the focusing polarity reversed to produce antineutrinos. Antineutrino studies are important for a complete test of LSND, which was primarily a $\bar{v}_{\mu} \rightarrow \bar{v}_{e}$ search.

\section{OSCILLATION RESULTS FROM MINIBOONE}

MiniBooNE has three previous appearance-mode oscillation results. The general technique is similar in all of them: events with a single electron-like Cherenkov ring are selected as charged-current quasielastic (CCQE) candidates. Cuts are designed to remove neutral-current $\pi^{0}$ events as well as fragments from neutrino interactions that occurred outside the detector ("dirt"). The reconstructed neutrino energy $E_{v}^{\mathrm{QE}}$ is computed assuming the process was $v N \rightarrow e N^{\prime}$ with the nucleon unobserved and the neutrino originating from the beam direction. Significant backgrounds are intrinsic $v_{e}$ in the beam, neutral-current $\pi^{0}$ and $\Delta \rightarrow N \gamma$, and dirt. Neutrinos and antineutrinos cannot be distinguished on an event-by-event basis.

In 2007, the main search for LSND-like oscillations in neutrino mode was published [2] based on $5.7 \times 10^{20}$ protons on target (POT). This result excluded a $C P$-conserving two-neutrino oscillation explanation for LSND at the 98\% confidence level. At the same time, an unexplained excess of $v_{e}$-like events below $475 \mathrm{MeV}$ was seen; further studies of this low-energy excess were published in 2009 [3].

In 2010, a search for $\bar{v}_{\mu} \rightarrow \bar{v}_{e}$ from $5.66 \times 10^{20}$ POT in antineutrino running mode was published [4]. That paper indicated an excess of $20.9 \pm 14.0 v_{e} / \bar{v}_{e}$ candidates in the $475-1250 \mathrm{MeV}$ range where MiniBooNE is most sensitive to LSND-like oscillations and contributions from the neutrino-mode low-energy excess are minimized. However, a likelihood-ratio fit to the energy distributions of both the $\bar{v}_{e}$ and $\bar{v}_{\mu}$ candidates preferred the oscillation hypothesis over the background-only hypothesis with $99.4 \%$ probability. In the low-energy region of $200-475 \mathrm{MeV}$, the data excess over the background prediction was $18.5 \pm 14.3$ events. Scaling the neutrino-mode low-energy excess by the expected neutrino contamination in the antineutrino flux gives a predicted low-energy excess of 12 events. 

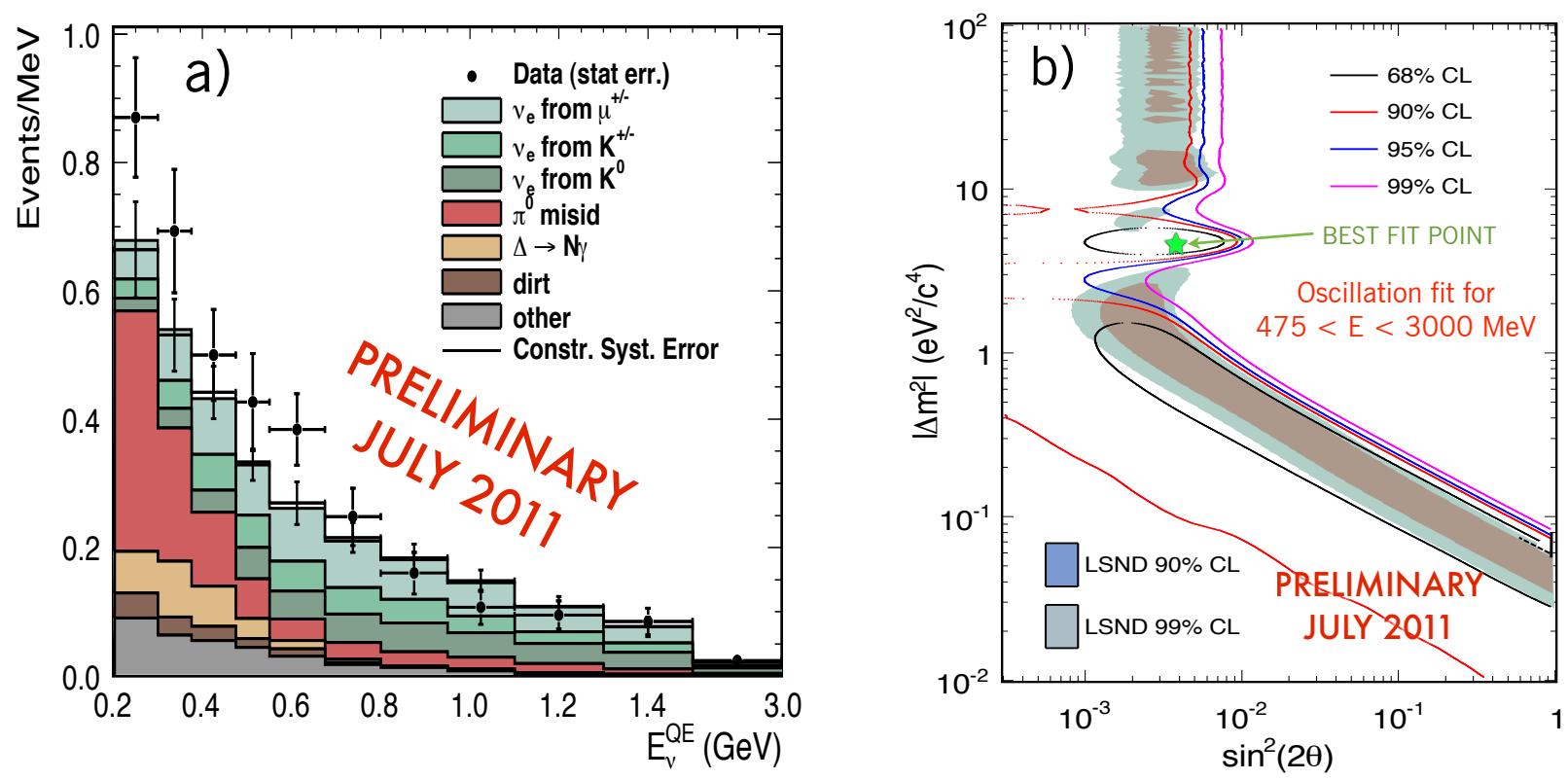

FIGURE 1. a) Reconstructed $E_{v}^{\mathrm{QE}}$ distribution for $\bar{v}_{e}$ candidates with data collected in antineutrino mode through May 2011. b) Oscillation fit contours for updated $\bar{v}_{e}$ appearance analysis. Energy range of fit is $475<E_{v}^{\mathrm{QE}}<3000 \mathrm{MeV}$. Results are preliminary.

TABLE 1. Preliminary predicted low-energy excess from scaling neutrino-mode excess by various factors. The value observed in data is $38.6 \pm 18.5$.

\begin{tabular}{lclc}
\hline \multicolumn{1}{c}{ Scaling basis } & Predicted excess & \multicolumn{1}{c}{ Scaling basis } & Predicted excess \\
\hline Total background & 50 & Neutrino contamination & 17 \\
$\Delta \rightarrow N \gamma$ decays & 39 & Dirt & 46 \\
Protons on target* & 165 & $K^{+}$in secondary beam & 67 \\
Neutral-current $\pi^{0}$ & 48 & Inclusive charged-current & 59 \\
\hline
\end{tabular}

* Excess would scale this way if due to neutral particles in secondary beam.

\section{UPDATED ANTINEUTRINO RESULTS}

In this section we present preliminary updates to the antineutrino oscillation search reported in Ref. [4] using $8.58 \times 10^{20}$ POT, using a nearly unchanged analysis. The most significant change is an improved constraint on kaondecay $v_{e}$ in the beam from the SciBooNE experiment [5], reducing the prediction for that background source by $3 \%$ and its error by a factor of three. Detector and beam monitoring have indicated no significant changes over the entire run period, and Kolmogorov-Smirnov tests of neutrino control samples are consistent with a constant event rate.

The reconstructed energy distribution of $\bar{v}_{e}$ candidates is shown in Fig. 1a. In the augmented data set, 168 events are observed in the $475-1250 \mathrm{MeV}$ region, corresponding to an excess of $16.3 \pm 19.4$ over predicted background. The excess in this region is thus reduced when the new data are added.

In the low-energy region, the excess has grown to $38.6 \pm 18.5$ events. We can compare this excess to predictions from scaling the observed neutrino-mode low-energy excess by various factors (see Table 1). This can exclude some possible phenomena as primary sources of the low-energy excess, though it should be noted that none of these processes can be scaled by a large enough factor to explain the low-energy excess without disagreeing strongly with control samples.

The primary test of LSND's result is the simultaneous fit to the $\bar{v}_{\mu}$ and $\bar{v}_{e}$ candidates in the $475<E_{v}^{\mathrm{QE}}<3000 \mathrm{MeV}$ range. The updated confidence-level contours are shown in Fig. 1b. The fit prefers the oscillation hypothesis to the background-only hypothesis at $91.1 \%$ confidence level. The best-fit point moved from the high-sin ${ }^{2}(2 \theta)$, low- 
$\Delta m^{2}$ region to the high- $\Delta m^{2}$ "island" solution, however the $\chi^{2}$ minimum is quite broad so this does not represent a significant change. In the signal bins, the $\chi^{2}$ probability is $14.9 \%$ for background-only and $35.5 \%$ for the oscillation fit. (These numbers were $0.5 \%$ and $10 \%$ before the statistics update.) The $68 \%$ confidence level contour still covers most of the LSND allowed region. This result is therefore still consistent with LSND, but the evidence for LSND-like oscillations is no longer as strong.

As in the published result, fits have been performed under other sets of assumptions. First, a fit to the entire energy range $\left(200<E_{v}^{\mathrm{QE}}<3000 \mathrm{MeV}\right)$ yields similar contours, with oscillations preferred with $97.6 \%$ probability. The fit $\chi^{2}$ probabilities for background-only and the best oscillation fit are $10.1 \%$ and $50.7 \%$ respectively. However, it should be noted that there is a large known neutrino contamination in antineutrino running (22\% of the $\bar{v}_{\mu}$ candidates and $44 \%$ of the total background to the $\bar{v}_{e}$ oscillation signal). A low-energy excess from neutrinos may be expected to contribute to the 200-475 MeV bins in this fit, but has not been subtracted because its origin and scaling are unknown. Therefore, this fit cannot be interpreted as a pure antineutrino physics result.

Another alternative fit is done with additional background subtraction, assuming that the low-energy excess contribution from neutrinos simply scales with the neutrino-induced event rate in each bin. This model's validity may be poor, however, if the excess is due to feed-down from misreconstructed higher-energy neutrinos with unobserved particles in the final state (since the neutrino spectrum differs between neutrino and antineutrino running). The fit under this background subtraction model prefers oscillations with $94.2 \%$ probability. The fit $\chi^{2}$ probabilities for background-only and the best oscillation fit are $28.3 \%$ and $76.5 \%$ respectively.

The contours and best-fit points for the fits to the full energy spectrum are available in the slides from this presentation.

\section{CONCLUSION AND NEXT STEPS}

MiniBooNE presents a preliminary update to its 2010 electron antineutrino appearance search with $52 \%$ more integrated flux. Adding the new data reduces the significance of the apparent LSND-like oscillation signal, and reveals a low-energy excess of $\bar{v}_{e}$ candidates similar to that observed in neutrino mode. In the higher-energy region, the new data are consistent with both LSND and a background-only hypothesis.

The experiment will continue to take data at least until the 2012 shutdown. The collaboration's goal is a total of $1.5 \times 10^{21}$ POT - nearly doubling the current data set. This statistically-limited result should benefit greatly from the additional data. If either our best-fit value or LSND's central value is correct, the expected exclusion of the backgroundonly hypothesis (relative to the oscillation fit) with a data set that large is at the $98-99 \%$ confidence level.

\section{ACKNOWLEDGMENTS}

The author and the MiniBooNE collaboration acknowledge the contributions of Fermi National Accelerator laboratory and support from the Department of Energy and the National Science Foundation.

\section{REFERENCES}

1. A. Aguilar-Arevalo et al., Phys. Rev. D64 112007 (2001).

2. A. Aguilar-Arevalo et al., Phys. Rev. Lett. 98231801 (2007).

3. A. Aguilar-Arevalo et al., Phys. Rev. Lett. 102101802 (2009).

4. A. Aguilar-Arevalo et al., Phys. Rev. Lett. 105181801 (2010).

5. G. Cheng et al., Phys. Rev. D84 012009 (2011). 